\title{
On the age of PSR B 1509-58
}

\author{
V. V. Gvaramadze $e^{1,2, \star}$ \\ 1 Abastumani Astrophysical Observatory, Georgian Academy of Sciences, A.Kazbegi ave. 2-a, Tbilisi 380060, \\ Georgia \\ 2 Sternberg State Astronomical Institute, Universitetskij Prospect 13, Moscow 119899, Russia
}

Received 26 April 1999 / Accepted 17 May 2001

\begin{abstract}
It is generally accepted that the PSR B 1509-58 is associated with the supernova remnant (SNR) MSH 15-52 (G 320.4-01.2). The spin-down age of the pulsar is $\simeq 1700$ years, while the size and the general appearance of the SNR suggest that this system is much older. A few possible explanations of this discrepancy have been put forward. We offer an alternative one and suggest that the high spin-down rate of the pulsar characterizes only a relatively short period of its (present) spin history, and that the enhanced braking torque is connected with the interaction between the pulsar's magnetosphere and the dense matter of a circumstellar clump (created during the late evolutionary stages of the supernova (SN) progenitor star). Our suggestion implies that the "true" age of PSR B 1509-58 could be much larger than the spin-down age, and therefore the SNR MSH 15-52 is a middle-aged remnant similar to the Vela SNR (G 263.9-3.3). We also suggest that the dense (neutral) gas of the circumstellar clump could be responsible for the enhanced neutral hydrogen absorption towards PSR B 1509-58, and that the optical emission of an optical counterpart for PSR B 1509-58 should rather be attributed to a bow shock around this pulsar than to the pulsar itself.
\end{abstract}

Key words. stars: neutron - pulsars: individual: B 1509-58 - ISM: bubbles - ISM: individual objects: MSH 15-52 - ISM: supernova remnants

\section{Introduction}

PSR B 1509-58 (Seward \& Harnden 1982, Manchester et al. 1982) is situated not far from the geometrical centre of the SNR MSH 15-52 (or G 320.4-01.2), and their association is beyond any doubt. However, this association causes a number of difficulties for the understanding and interpretation of the observational data. The problem is that the size and the general appearance of the SNR suggest that it should be much older than it follows from the pulsar age estimates.

It is usually assumed that the rotational frequency $\Omega$ of a pulsar decreases according to the relation $\dot{\Omega}=-K \Omega^{n}$, where $K$ depends upon the physics of the slow-down mechanism, and $n=\Omega \ddot{\Omega} / \dot{\Omega}^{2}$ is the braking index. Assuming constant $K$ and $n$, and provided that the initial spin period $P_{\mathrm{i}}$ of the pulsar was much smaller than the current period $P=2 \pi / \Omega$, one can estimate the characteristic spin-down age $\tau=P /(n-1) \dot{P}$. For $P \simeq 0.15 \mathrm{~s}$, $\dot{P} \simeq 1.49 \times 10^{-12} \mathrm{~s} \mathrm{~s}^{-1}$ and $n=2.84$ (Weisskopf et al. 1983; Manchester et al. 1985; Kaspi et al. 1994), one derives an age of PSR $\mathrm{B} 1509-58$ of $\simeq 1700$ years, i.e. it is

* Address for correspondence: Krasin str. 19, ap. 81, Moscow 123056, Russia.

e-mail: vgvaram@mx.iki.rssi.ru nearly as young as the Crab pulsar. The spin-down age could be even less by a factor of $\left[1-\left(P_{\mathrm{i}} / P\right)^{n-1}\right]^{-1}$, if the pulsar was born with $P_{\mathrm{i}}$ of $\simeq 0.1 \mathrm{~s}$ (see e.g. Spruit \& Phinney 1998). These estimates are at odds with the age estimates for MSH 15-52 (Seward et al. 1983; van den Berg \& Kamper 1984; Kamper et al. 1995), which show that the SNR is a much older object.

To reconcile the ages of the pulsar and the SNR, Seward et al. (1983) considered two possibilities: 1) MSH 15-52 is a young SNR, and 2) PSR B 1509-58 is an old pulsar. The first one implies (in the framework of the Sedov-Taylor model) that the SN explosion was very energetic and occured in a tenuous medium (see also Bhattacharya 1990). This point of view is generally accepted (e.g. Kaspi et al. 1994; Greiveldinger et al. 1995; Trussoni et al. 1996; Gaenzler et al. 1999). The second possibility implies that $\tau$ is at least few times shorter than the "true" age (Seward et al. 1983). This possibility was re-examinated by Blandford \& Romani (1988). Assuming that the pulsar spin-down is mostly due to the electromagnetic torque, they suggested that the torque grew within the last $\simeq 10^{3}$ years due to the growth of the pulsar's magnetic field (see also Muslimov \& Page 1996). In this case, the coefficient $K$ is an increasing function of time and therefore the "true" age of the pulsar could be as large as 
follows from the age estimates for the SNR. In this paper we offer an alternative explanation for the increase of the braking torque (Sect. 2), viz. we suggest that it could be episodically enhanced due to the interaction of the pulsar's magnetosphere with dense clumps of circumstellar matter (Sect. 3). Section 4 deals with some issues related to our suggestion.

\section{The spin-down of PSR B 1509-58}

It is known that the electromagnetic torque acting on a rotating, magnetized body (e.g. a neutron star) immersed in a plasma is enhanced as compared with the torque in vacuum (Ginzburg 1971, see also Istomin 1994). It was mentioned by Istomin (1994) that for the increase of the slow-down torque of a pulsar it is suffient to have a dense plasma in the vicinity of the light cylinder, since just in this region the pulsar loses its rotational energy due to the acceleration of particles. The particles of the ambient medium penetrating into the region of the light cylinder are accelerated there to velocities comparable with the speed of light and then leave this region (Istomin 1994). This presumably equatorial outflow (cf. Brinkmann et al. 1985; King \& Cominsky 1994) carries away the pulsar's angular momentum and is responsible for the enhanced braking of the pulsar. We suggest (see also Gvaramadze 1999a; cf. Yusifov et al. 1995; Istomin \& Komberg 2000) that just this effect is responsible for the present high spindown rate of PSR B 1509-58, i.e. that the pulsar loses its rotational energy mainly due to the acceleration of protons of the ambient medium arriving at the light surface at the rate $\dot{M}$ :

$|\dot{E}|=|I \Omega \dot{\Omega}|=\gamma_{\mathrm{p}} \dot{M} c^{2}$

where $I \simeq 10^{45} \mathrm{~g} \mathrm{~cm}^{2}$ is the moment of inertia of the pulsar, $c$ is the speed of light, and $\gamma_{p}$ is the Lorentz factor of accelerated protons. For $\Omega=41.89 \mathrm{~s}^{-1}$ and $\dot{\Omega}=-4.25 \times 10^{-10} \mathrm{~s}^{-2}$ (e.g. Kaspi et al. 1994), and $\gamma_{\mathrm{p}} \simeq 1$ (Istomin 1994), one has $\dot{M} \simeq 2.2 \times 10^{16} \mathrm{~g} \mathrm{~s}^{-1}$. We also suggest that the pulsar moves through the inhomogeneous ambient medium and episodically plunges into clumps of dense matter. If the density of clumps is sufficiently high (see Sect. 3), one can expect a temporary increase of $\dot{\Omega}$ every time the pulsar travels through a clump (we believe that just this situation takes place now). The corresponding spin-down age will be less than estimated when the pulsar moves through the low-density interclump medium.

It is clear that the presence of radio emission of the pulsar means that the ambient medium does not penetrate far beyond the light surface. Assuming that the ram pressure of the accreting medium is equal to the magnetic pressure at the light surface (cf. King \& Cominsky 1994), one has an estimate of the surface magnetic field of the pulsar $^{1}$ :

$B_{*}=\left[2\left(2 G M_{*}\right)^{1 / 2} \dot{M} r_{\mathrm{L}}^{7 / 2} / r_{*}^{6}\right]^{1 / 2} \simeq 2.9 \times 10^{12} \mathrm{G}$,

where $G$ is the gravitational constant, $M_{*}=1.4 M_{\odot}$ and $r_{*}=10^{6} \mathrm{~cm}$ are the mass and the radius of the pulsar, and $r_{\mathrm{L}}=c / \Omega \simeq 7.1 \times 10^{8} \mathrm{~cm}$ is the radius of the light cylinder; for simplicity we assumed that the pulsar magnetic field is dipolar. Given this value of $B_{*}$, one can estimate the "vacuum" values of $\dot{\Omega}$ and $\tau: \dot{\Omega}_{0}=-2 \mu^{2} \Omega^{3} / 3 c^{3} I \simeq-1.36 \times$ $10^{-11} \mathrm{~s}^{-2}$, where $\mu=B_{*} r_{*}^{3}$ is the magnetic momentum of the pulsar, and $\tau_{0} \leq-\Omega / 2 \dot{\Omega}_{0} \simeq 4.9 \times 10^{4} \mathrm{yr} \simeq 30 \tau$ (here we assumed that the "vacuum" braking index is equal to $3)$. This means that the "true" age of the pulsar could be as large as $\tau_{0}$ (provided that $P_{\mathrm{i}} \ll P$ ) and that the SNR MSH 15-52 could be a middle-aged remnant similar to the Vela SNR (G 263.9-3.3).

\section{Dense clumps in the central part of SNR MSH 15-52}

We suggest that PSR B 1509-58 and SNR MSH 15-52 are the remnants of the SN explosion of a massive star (Gvaramadze 1999b). In this case, the structure of MSH 15-52 could be determined by the interaction of the SN blast wave with the ambient medium reprocessed by the joint action of the ionizing emission and stellar wind of the SN progenitor star (McKee et al. 1984; Shull et al. 1985; Ciotti \& D'Ercole 1989; Chevalier \& Liang 1989; Franco et al. 1991; D'Ercole 1992; Gvaramadze 1999b,c, 2000a). The outer shell of the SNR could arise due to the abrupt deceleration of the SN blast wave after it encounters the density jump at the edge of the bubble created by the fast stellar wind during the main-sequence or the Wolf-Rayet (WR) stages. On the other hand, some structures in the central part of the $\mathrm{SNR}^{2}$ could be attributed to the interaction of the SN blast wave with the circumstellar material lost during the late evolutionary stages of the SN progenitor star [this is the material that determines the appearance of young type II SNRs, e.g. SN 1987A (e.g. McCray 1993) or Cas A (e.g. Garcia-Segura et al. 1996; Borkowski et al. 1996)]. During the red supergiant (RSG) stage a massive star loses a significant part (about two thirds) of its mass in the form of a slow, dense wind. This matter occupies a compact region with a characteristic radius of few parsecs (the high-pressure gas in the main-sequence bubble significantly affects the spreading of this region, Chevalier \& Emmering 1989; D'Ercole 1992). Before the SN exploded, the progenitor star (of mass $\left.>15-20 M_{\odot}\right)$ becomes for a short time a WR star

\footnotetext{
1 Note that this estimate is about ten times smaller than what follows from the usual dipole formula (Weisskopf et al. 1983).

2 Note that these structures could be significantly offset from the geometrical centre of a middle-aged SNR due to the proper motion of the SN progenitor star (Gvaramadze 1999b, $2000 \mathrm{a}, \mathrm{b})$.
} 
(e.g. Vanbeveren et al. 1998). At this stage, the fast stellar wind sweeps up the slow RSG wind and creates a low-density cavity surrounded by a shell of swept-up circumstellar matter. The shell expands with a nearly constant velocity $v_{\mathrm{sh}} \simeq\left(\dot{M}_{\mathrm{WR}} v_{\mathrm{WR}}^{2} v_{\mathrm{RSG}} / 3 \dot{M}_{\mathrm{RSG}}\right)^{1 / 3}$, where $\dot{M}_{\mathrm{WR}}, \dot{M}_{\mathrm{RSG}}$ and $v_{\mathrm{WR}}, v_{\mathrm{RSG}}$ are, correspondingly, the mass-loss rates and wind velocities during the WR and RSG stages (e.g. Dyson 1981), until it catches up the shell separating the RSG wind from the main-sequence bubble. For parameters typical for RSG and WR winds, one has $v_{\mathrm{sh}} \simeq 100-200 \mathrm{~km} \mathrm{~s}^{-1}$. The interaction of two circumstellar shells results in Rayleigh-Taylor and other dynamical instabilities, whose development is accompanied by the formation of dense clumps moving with radial velocities of $\simeq v_{\text {sh }}$ (Garcia-Segura et al. 1996). The dense clumps could originate much closer to the SN progenitor star due to the stellar wind acceleration during the transition from the RSG to the WR stage (Brighenti \& D'Ercole 1997). The number density of clumps is $\geq 10^{5} \mathrm{~cm}^{-3}$ provided they are not fully ionized and were able to cool to a temperature of $\leq 10^{2} \mathrm{~K}$ (Brighenti \& D'Ercole 1997). Direct evidence of the existence of high-density clumps close to the SN explosion sites follows from observations of young SNRs. For example, the optically emitting gas of quasi-stationary flocculi in Cas A is characterized by a density of $\simeq 10^{4} \mathrm{~cm}^{-3}$ and a temperature of $\geq 10^{4} \mathrm{~K}$ (e.g. Lozinskaya 1992). Assuming that the optical emission of a floccule comes from an ionized "atmosphere" around the neutral core, one can estimate the density of the core to be $\geq 10^{6} \mathrm{~cm}^{-3}$, provided that the temperature of the core is $\leq 10^{2} \mathrm{~K}$. Similar estimates could also be derived from observations of the optical ring around SN 1987A, the inner ionized "skin" of which has nearly the same parameters (e.g. Plait et al. 1995) as the optically emitting gas of flocculi in Cas A, or from observations of some other young SNRs (e.g. Chugai 1993; Chugai \& Danziger 1994). The radial velocity of flocculi in Cas A ranges from $\simeq 80$ to $\simeq 400 \mathrm{~km} \mathrm{~s}^{-1}$ (e.g. Lozinskaya 1992).

Initially, the new-born pulsar moves through the lowdensity cavity created by the fast wind of the presupernova star until it plunges into the first dense clump on its way. This happens at the moment $t \sim r_{\text {cav }} / v_{*}$, where $r_{\text {cav }} \simeq 1-2 \mathrm{pc}$ is the radius of the cavity, $v_{*}=v_{\mathrm{p}}-v_{\mathrm{cl}}$, $v_{\mathrm{p}}$ and $v_{\mathrm{cl}}$ are respectively the velocities of the pulsar and the clump. For $v_{\mathrm{p}} \simeq 150 \mathrm{~km} \mathrm{~s}^{-1}$ and $v_{\mathrm{cl}} \simeq 100 \mathrm{~km} \mathrm{~s}^{-1}$, one has $t \simeq(2-4) \times 10^{4}$ years $^{3}$. Let us assume that all matter captured inside the accretion radius $r_{\text {acc }}=2 G M_{*} / v_{*}^{2}$ $\left(v_{*} \gg c_{\mathrm{S}}\right.$, where $c_{\mathrm{S}}$ is the sound speed in the cold, dense circumstellar clump) of the pulsar moving through the clump

\footnotetext{
${ }^{3}$ No proper motion for PSR B 1509-58 has yet been detected (Kaspi et al. 1994; Gaensler et al. 1999), though the recent finding of the candidate optical counterpart of this pulsar (Caraveo et al. 1994, see also Mignami et al. 1998 and Sect. 4) allows one to hope that it will be detected in due course. For the distance to the pulsar of $5 d_{5} \mathrm{kpc}$ (e.g. Gaensler et al. 1999), a velocity of $150 \mathrm{~km} \mathrm{~s}^{-1}$ corresponds to a proper motion of $\simeq 6 \mathrm{mas} \mathrm{yr}^{-1}$; this is much smaller than the upper limits on the pulsar's proper motion given by Kaspi et al. (1994) and Gaensler et al. (1999).
}

penetrates into the region of the light cylinder, where it is accelerated to relativistic velocities and then leaves this region in the form of equatorial outflow (cf. Istomin 1994; King \& Cominsky 1994). The rate at which the ambient medium arrives at the light cylinder could be estimated as

$\dot{M}=\pi r_{\mathrm{acc}}^{2} n_{\mathrm{cl}} m_{\mathrm{p}} v_{*}$,

where $n_{\mathrm{cl}}$ is the number density of the clump, and $m_{\mathrm{p}}$ is the mass of a proton. If the pulsar braking is indeed mainly due to the acceleration of circumstellar protons arriving at the light surface, then one obtains from (1) and (2) that $n_{\mathrm{cl}} \simeq 3.8 \times 10^{6} v_{*, 50}^{2} \mathrm{~cm}^{-3}$, where $v_{*, 50}=v_{*} / 50 \mathrm{~km} \mathrm{~s}^{-1}$. The time it takes for PSR B 1509-58 to cross the clump, $t^{\prime} \simeq l_{\mathrm{cl}} / v_{*}$, where $l_{\mathrm{cl}}$ is the characteristic size of clumps, should be larger than the time since the pulsar discovery, i.e. $t^{\prime}>30$ years. This requirement results in $l_{\mathrm{cl}} \geq 5 \times$ $10^{15} v_{*, 50} \mathrm{~cm}$ and $M_{\mathrm{cl}}>0.0002 v_{*, 50}^{3} M_{\odot}$, where $M_{\mathrm{cl}}$ is the characteristic mass of clumps. For the SN progenitor star of mass $\geq 15 M_{\odot}$, the mass of the circumstellar gas (i.e. the matter lost during the RSG stage) is about $10 M_{\odot}$, and the number of clumps is $<5 \times 10^{4}$. For the current radius of the region occupied by circumstellar matter of about 10 pc (Gvaramadze 1999b), one finds a covering factor of the clumpy circumstellar material (i.e. the fraction of the sphere occupied by clumps $\simeq 10^{-4}$.

For accretion to occur, the standoff radius $r_{\mathrm{s}}$ of the bow shock (formed by the outflow of relativistic particles) should be less than $r_{\text {acc }}$. For the spherically symmetric outflow, one has $r_{\mathrm{s}}=\left(\alpha|\dot{E}| / 4 \pi n_{\mathrm{cl}} m_{\mathrm{p}} c v_{*}^{2}\right)^{1 / 2}<r_{\mathrm{acc}}=$ $2 G M_{*} / v_{*}^{2}$, where we assume that only a fraction $\alpha<1$ of the spin-down luminosity $|\dot{E}|$ is transferred to the ambient medium (cf. Kochanek 1993). This condition can be re-written as $\alpha<\left(\gamma_{\mathrm{p}} / 4\right)^{-1} v_{*} / c \simeq 10^{-3} \gamma_{\mathrm{p}}^{-1} v_{*, 50}$ (cf. Kochanek 1993; Manchester et al. 1995), i.e. $\alpha$ should be much smaller than the usually adopted value of $\simeq 1$ (e.g. Kulkarni \& Hester 1988; Cordes et al. 1993). Weak coupling $(\alpha \ll 1)$ of the pulsar wind with the ambient medium is consistent with an outflow composed of highly relativistic particles (e.g. Kochanek 1993 and references therein). Alternatively, if the outflow of relativistic particles is confined to the vicinity of the rotational equatorial plane, one can expect that the ambient matter accretes onto the pulsar's magnetosphere along the polar directions. Another possibility is that the ambient matter penetrates in the pulsar wind bubble through instabilities in the bow shock front. In the latter both cases $r_{\mathrm{s}}$ could be larger than $r_{\mathrm{acc}}$, and one can adopt $\alpha \simeq 1$ (see next section).

\section{Discussion}

We now discuss some consequences of our proposal that the braking of PSR B 1509-58 is mostly due to the interaction of the pulsar's magnetosphere with the dense matter of a circumstellar clump.

First, we consider the contribution of the circumstellar matter to the neutral hydrogen absorption toward the 
pulsar. The low covering factor of clumps (see Sect. 3) implies that this contribution is

$N_{\mathrm{cl}}=\int_{r_{\mathrm{L}}}^{r_{\mathrm{acc}}} n_{\mathrm{ff}}(r) \mathrm{d} r+n_{\mathrm{cl}}\left(r_{\mathrm{cl}}-r_{\mathrm{acc}}\right)$

where $n_{\mathrm{ff}}(r)=\dot{M} / 4 \pi m_{\mathrm{p}} r^{2} v_{\mathrm{ff}}$ is the number density of the infalling gas captured inside the accretion radius, $v_{\mathrm{ff}}=$ $\left(2 G M_{*} / r\right)^{1 / 2}$ is the free-fall velocity, $r_{\mathrm{cl}}$ is the line of sight thickness of the dense neutral gas located between the pulsar and the observer $\left(r_{\mathrm{cl}} \leq l_{\mathrm{cl}}\right)$.

It was pointed out by Strom (1994) that a ROSAT observation of the SNR MSH 15-52 indicates a larger absorption towards PSR B 1509-58 than seen from the bright northwest part of the SNR's shell (known as RCW 89). The comparison of neutral hydrogen absorption data (see Greiveldinger et al. 1995; Trussoni et al. 1996; Tamura et al. 1996; Marsden et al. 1997; Rots et al. 1998) shows that the excess of absorption towards the pulsar could be as large as $\simeq(1-5) \times 10^{22} \mathrm{~cm}^{-2}$. This discrepancy could be interpreted as a sign that the pulsar is more distant than the SNR, and therefore that these two objects are not physically associated with each other (Strom 1994). It is also possible that "the spectral analysis is not detailed enough to provide the correct parameters" (Trussoni et al. 1996). Another possibility is that the H I column density distribution is really inhomogeneous across the SNR (cf. Trussoni et al. 1996). We favour the last possibility and suggest that the excess of absorption towards PSR B 150958 is due to the dense neutral gas around the pulsar. One can use Eq. (3) to set an upper limit on $r_{\mathrm{cl}}$. For the parameters adopted above, and assuming that $N_{\mathrm{cl}} \leq$ (1$5) \times 10^{22} \mathrm{~cm}^{-2}$, one has $r_{\mathrm{cl}} \leq(0.2-1.2) \times 10^{16} v_{*, 50}^{-3} \mathrm{~cm}$. This estimate shows that if our explanation of the age discrepancy is correct, then one might expect that in the near future (i.e. after a lapse of $\simeq r_{\mathrm{cl}} / v_{*, \|}$, where $v_{*, \|}$ is the line of sight component of $v_{*}$ ) the first derivative of the pulsar's spin period will suffer a significant decrease.

Second, let us discuss the candidate optical counterpart for PSR B 1509-58 proposed by Caraveo et al. (1994). Caraveo et al. pointed out that the luminosity of the optical counterpart $\left(L_{\mathrm{V}} \sim 6.5 \times 10^{32} d_{5}^{2} \mathrm{ergs} \mathrm{s}^{-1}\right)$ exceeds by a few orders of magnitude the value derived for magnetospheric optical emission of young pulsars (Pacini 1971). This fact together with the negative result of searching of optical pulsations at the radio period led to the conclusion that the proposed identification could be erroneous (Mignami et al. 1998; see also Shearer et al. 1998; Chakrabarti \& Kaspi 1998). We suggest, however, that the observed optical emission should rather be attributed to the bow shock around the pulsar than to the pulsar itself. This suggestion is supported by the estimate of the total luminosity of the bow shock, $L_{\mathrm{T}} \simeq S n_{\mathrm{cl}} v_{*}\left(m_{\mathrm{p}} v_{*}^{2} / 2\right)$, where $S \simeq \pi r_{\mathrm{b}}^{2}$ and $r_{\mathrm{b}} \simeq 3^{1 / 2} r_{\mathrm{s}}$ are the area and the characteristic radius of the bow shock, respectively. For the adopted parameters $^{4}$ this gives $L_{\mathrm{T}} \simeq \alpha \times 10^{33} \mathrm{ergs} \mathrm{s}^{-1}$, i.e. $L_{\mathrm{V}} \simeq L_{\mathrm{T}}$ for $\alpha \simeq 1$. It is obvious that if our suggestion is correct, there can be no correspondence between the observed luminosity and the luminosity expected from the results of Pacini (1971). The optical pulsations should be absent as well.

To conclude, we point out a curious coincidence of the accretion rate derived in Sect. 2 with accretion rates required in accretion-based models to explain high spindown rates of anomalous X-ray pulsars and soft gammaray repeaters (e.g. Mereghetti \& Stella 1995; Ghosh et al. 1997; Chatterjee et al. 2000; Alpar 2000). This coincidence allows us to believe (Gvaramadze 1999a, 2000b) that these objects could lose a significant part of their rotational energy due to the process discussed in this paper, and that their "true" ages could be much larger than the respective characteristic spin-down ones.

Acknowledgements. I am grateful to N. D'Amico and A. D'Ercole for discussions, to D. Page (the referee) for comments, and to J. K. Katgert-Merkelijn (the Deputy Editor) for carefully reading the manuscript. This work was partially supported by NPS.

\section{References}

Alpar, M. A. 2000 [astro-ph/0005211]

Bhattacharya, D. 1990, JA\&A, 11, 125

Blandford, R. D., \& Romani, R. W. 1988, MNRAS, 234, 57p

Borkowski, K. J., Szymkowiak, A. E., Blondin, J. M., \& Sarazin, C. L. 1996, ApJ, 466, 866

Brighenti, F., \& D'Ercole, A. 1997, MNRAS, 285, 387

Brinkmann, W., Aschenbach, B., \& Langmeier, A. 1985, Nature, 313, 662

Caraveo, P. A., Mereghetti, S., \& Bignami, G. F. 1994, ApJ, 423, L125

Chakrabarty, D., \& Kaspi, V. M. 1998, ApJ, 498, L37

Chatterjee, P., Hernquist, L., \& Narayan, R. 2000, ApJ, 534, 373

Chevalier, R. A., \& Emmering, R. T. 1989, ApJ, 342, L75

Chevalier, R. A., \& Liang, E. P. 1989, ApJ, 344, 332

Chugai, N. N. 1993, ApJ, 414, L101

Chugai, N. N., \& Danziger, I. J. 1994, MNRAS, 268, 173

Ciotti, L., \& D'Ercole, A. 1989, A\&A, 215, 347

Cordes, J. M., Romani, R. W., \& Lundgren, S. C. 1993, Nature, 362,133

D'Ercole, A. 1992, MNRAS, 255, 572

Dyson, J. E. 1981, in Investigating the Universe, ed. F. D. Kahn (Reidel, Dordrecht), 125

Franco, J., Tenorio-Tagle, G., Bodenheimer, P., \& Różyczka, M. 1991, PASP, 103, 803

Gaensler, B. M., Brazier, K. T. S., Manchester, R. N., Johnston, S., \& Green, A. J. 1999, MNRAS, 305, 724

Garsia-Segura, G., Langer, N., \& Mac Low, M.-M. 1996, A\&A, 316, 133

Ghosh, P., Angelini, L., \& White, N. E. 1997, ApJ, 478, 713

${ }^{4}$ Note that shocks in the range $\left(v_{\mathrm{s}} \geq 40 \mathrm{~km} \mathrm{~s}^{-1}\right.$, where $v_{\mathrm{s}}$ is the shock velocity $\left.\left(\simeq v_{*}\right), 10^{6} \mathrm{~cm}^{-3} \leq n_{\mathrm{cl}} \leq 10^{8} \mathrm{~cm}^{-3}\right)$ produce strong $22 \mathrm{GHz} \mathrm{H} \mathrm{H}_{2} \mathrm{O}$ masers (see Fig. 3 of Hollenbach 1997). The possible detection of $\mathrm{H}_{2} \mathrm{O}$ masers towards PSR B 1509-58 will lend strong support to the suggestions of this paper. 
Ginzburg, V. L. 1971, Sov. Phys. Usp., 14, 83

Greiveldinger, C., Caucino, S., Massaglia, S., Ögelman, H., \& Trussoni, E. 1995, ApJ, 454, 855

Gvaramadze, V. V. 1999a, in Astrophysics on the Boundary of Centuries, ed. N. S. Kardashev, R. D. Dagkesamansky, \& Yu. A. Kovalev (ASC, Moscow, in Russian), 163

Gvaramadze, V. V. 1999b, Odessa Astron. Publ., 12, 117 [or astro-ph/9912512]

Gvaramadze, V. V. 1999c, A\&A, 352, 712

Gvaramadze, V. V. 2000a, submitted to MNRAS [astro-ph/0005572]

Gvaramadze, V. V. 2000b, in Abstract Book of the Joint European and National Astronomical Meeting, JENAM2000 (held in Moscow, Russia, May 29-June 3, 2000), 95

Hollenbach, D. 1997, in Herbig-Haro Flows and the Birth of Low Mass Stars, ed. B. Reipurth, \& C. Bertout (Kluwer, Dordrecht), 181

Istomin, Ya. N. 1994, A\&A, 283, 85

Istomin, Ya. N., \& Komberg, B. V. 2000, ARep, 44, 754

Kamper, K. W., van den Bergh, S., \& Westerlund, B. 1995, AAS, 186.3705

Kaspi, V. M., Manchester, R. N., Siegman, B., Johnston, S., \& Lyne, A. G. 1994, ApJ, 422, L83

King, A., \& Cominsky, A. 1994, ApJ, 435, 411

Kochanek, C. S. 1993, ApJ, 406, 638

Kulkarni, S. R., \& Hester, J. J. 1988, Nature, 335, 801

Lozinskaya, T. A. 1992, Supernovae and Stellar Winds in the Interstellar Medium (New York, AIP)

Manchester, R. N., Tuohy, I. R., \& D'Amico, N. 1982, ApJ, 262, L31

Manchester, R. N., Durdin, J. M., \& Newton, L. M. 1985, Nature, 313, 374

Manchester, R. N., Johnston, S., Lyne, A. G., et al. 1995, ApJ, 445, L137

Marsden, D., Blanco, P. R., Gruber, D. E., et al. 1997, ApJ,
491, L39

McKee, C. F., Van Buren, D., \& Lazareff, R. 1984, ApJ, 278, $\mathrm{L} 115$

McCray, R. 1993, ARA\&A, 31, 175

Mereghetti, S., \& Stella, L. 1995, ApJ, 442, L17

Mignani, R. P., Mereghetti, S., Gouiffes, C., \& Caraveo, P. A. 1998, The Messenger, 94, 25

Muslimov, A., \& Page, D. 1996, ApJ, 458, 347

Pacini, F. 1971, ApJ, 163, L17

Plait, P. C., Lundqvist, P., Chevalier, R. A., \& Kirshner, R. P. 1995, ApJ, 439, 730

Rots, A. H., Jahoda, K., Macomb, D. J., et al. 1998, ApJ, 501, 749

Seward, F. D., \& Harnden, F. R. 1982, ApJ, 256, L45

Seward, F. D., Harnden, F. R., Murdin, P., \& Clark, D. H. 1983, ApJ, 267, 698

Shearer, A., O'Sullivan, C. M. M., Golden, A., et al. 1998, A\&A, 333, L16

Shull, P., Dyson, J. E., Kahn, F. D., \& West, K. A. 1985, MNRAS, 212, 799

Spruit, H., \& Phinney, E. S. 1998, Nature, 393, 139

Strom, R. G. 1994, MNRAS, 268, L5

Tamura, K., Kawai, N., Yoshida, A., \& Brinkmann, W. 1996, PASJ, 48, L33

Trussoni, E., Massaglia, S., Caucino, S., Brinkmann, W., \& Aschenbach, B. 1996, A\&A, 306, 581

Vanbeveren, D., De Loore, C., \& Van Rensbergen, W. 1998, A\&AR, 9, 63

van den Berg., \& Kamper K. 1984, ApJ, 280, L51

Weisskopf, M. C., Elsner, R. F., Darbro, W., et al. 1983, ApJ, 267, 711

Yusifov, I. M., Alpar, M. A., Gök, F., \& Hüseyinov, O. H. 1995, in The Lives of the Neutron Stars, ed. M. A. Alpar, Ü. Kiziloğlu, \& J. van Paradijs (Kluwer, Dordrecht), 201 